\title{
Molecular Cloning and Characterization of a cDNA Encoding Sedoheptulose-1,7-bisphosphatase from Mulberry (Morus alba var. multicaulis)
}

\author{
By X. JI ${ }^{1)}$, Y. $\mathrm{GAI}^{2)}$, J. $\mathrm{MA}^{1)}$, C. $\mathrm{ZHENG}^{2)}$ and $\left.\mathrm{Z} . \mathrm{MU}^{1)}{ }^{1,}{ }^{*}\right)$
}

(Received $8^{\text {th }}$ December 2006)

\begin{abstract}
A full-length cDNA encoding sedoheptulose-1,7-bisphosphatase (SBPase; EC 3.1.3.37) was cloned from mulberry (Morus alba var. multicaulis) by rapid amplification of cDNA ends (RACE). The cDNA consisted of 1,527 nucleotides with an open reading frame (ORF) of 1,179 nucleotides encoding a 393 amino acid protein of approximately $42.6 \mathrm{kDa}$. Sequence comparison analysis showed that mulberry SBPase (MSBPase) had high homology to other plant counterparts. Phylogenetic and molecular evolutionary analysis revealed that MSBPase fell into plant SBPase group. Moreover, SBPase and fructose-1,6-bisphosphatase (FBPase; EC 3.1.3.11) shared $28-32 \%$ identical residues, suggesting that the two enzymes originated from the same evolution branch. Molecular modeling indicated that each subunit of MSBPase was composed of $\alpha$-helices and $\beta$-sheets joined by turns and loops, and folded into a structure of hexahedron shape which was very similar to FBPase.
\end{abstract}

Key words: sedoheptulose-1,7-bisphosphatase, fructose-1,6-bisphosphatase, cDNA cloning, mulberry.

\section{Introduction}

Sedoheptulose-1,7-bisphosphatase (SBPase) is a key enzyme in the regenerative phase of Calvin cycle, which is the primary pathway of carbon fixation in the chloroplast stroma (ANJA et al., 2002). SBPase catalyses the irreversible dephosphorylation of sedoheptulose-1,7-bisphosphate to sedoheptulose-7-phosphate (CHRISTINE et al., 1999). This reaction is essentially irreversible and commits intermediates to the regenerative part of the Calvin cycle, so as to it competes for the substrates with the starch synthetic pathway. Two enzymes, SBPase and FBPase, catalyse the irreversible reactions in the branched region where the intermediates may leave the cycle, and for this reason may have particular roles in regulating carbon portioning to sucrose and starch (WoOdrow and BerRY, 1988; GEIGER and SERVAITES, 1995). It is proposed that SBPase plays an important role in regulating the flow of intermediates through the Calvin cycle (PETterson and RYde-PETterson, 1989; Poolman et al., 2000). SBPase is a nuclear-encoded enzyme synthesized as a precursor of 393 amino acids

1) College of Forestry, Shandong Agricultural University, Taian, Shandong, 271018, People's Republic of China.

2) State Key Laboratory of Crop Biology, Shandong Agricultural University, Taian, Shangdong, 271018, People's Republic of China.

*) Corresponding Author: ZHIMEI Mu, College of Forestry, Shandong Agricultural University, Taian, Shandong, 271018, People's Republic of China. Tel: +86-538-8241315, Fax: +86538-8249131. E-mail: gyp@sdau.edu.cn with a transit peptide which directs the enzyme to the chloroplast. Biochemical studies including the purification of the SBPase protein from a number of species show that the SBPase is dimeric and comprises two identical subunits of molecular weight $35-38 \mathrm{kDa}$ (CADET et al., 1987; CHRISTINE et al., 1999).

Mulberry (Morus alba L.) is a perennial woody plant of considerable economic importance because of its foliage, which constitutes the chief food for the mulberry silkworm (Bombyx mori L.) (JAIN et al., 2000). We are interested in mulberry SBPase because of its pivotal role in the photosynthesis which products are used to synthesize a variety of metabolites beneficial to silkworm growth and development. Here, we described the isolation and nucleotide sequence analysis of the MSBPase cDNA from mulberry and compared its structure with SBPases from other plant species.

\section{Materials and Methods}

Plant materials and chemicals

Mulberry (Morus alba var. multicaulis) leaves were frozen immediately in liquid nitrogen and stored at

Table 1. - Oligo nucleotide primers used for RT-PCR and RACE-PCR.

\begin{tabular}{|c|c|}
\hline Name & Sequence $\left(5^{\prime}---3^{\prime}\right)$ \\
\hline $\mathrm{T} 15$ & GACTCTAGACGACATCGA(T) \\
\hline ST & GACTCTAGACGACATCGA \\
\hline SBP-1 & TTY GGN GAY GAR CAR YTN GC \\
\hline $\mathrm{SBP}^{\mathrm{P}-2}$ & TG RTT NAC RTC NGG NAC CAT \\
\hline $3 \mathrm{sw}$ & TCCTGGAAACTTGAGAGCCACT \\
\hline $3 \mathrm{sN}$ & CITCGAGACACACIGGAGGAAG \\
\hline$G_{\mathrm{H}} L_{\mathrm{S}}$ & GGCCACGGGTCGACTAGTAC $(\mathrm{G})_{1 \mathrm{~B}}$ \\
\hline $\mathrm{GT}$ & GGCCACGCOTCGACTAGTAC \\
\hline $5 s w$ & AGC CAC ACT GAA TCC ACS TT \\
\hline $\operatorname{ssN}$ & GGA ACT TCC TEG GAA CAA GCA \\
\hline
\end{tabular}


$-80^{\circ} \mathrm{C} . \mathrm{Ex} \mathrm{Taq}^{\mathrm{TM}}$, pMD18-T vector, T4 DNA ligase, DNA Gel Extraction Kit, 100 bp DNA Ladder, Reverse Transcriptase M-MLV, and Terminal Deoxynucleotidyl Transferase were all from Takara Biotechnology Co., Ltd. (Dalian, China). The degenerate and specific primers used in PCR were synthesized by Shanghai Invitrogen Biological Engineering Technology and Service Co., Ltd. (Shanghai, China). The primers used in this study were all listed in Table 1.

\section{RNA isolation and cDNA synthesis}

Mulberry leaves were ground to fine powder in liquid nitrogen and total RNA was extracted by means of the hexadecyltrimethylammonium bromide (CTAB) method (CHANG et al., 1993). To synthesize the first strand cDNA, $2 \mu \mathrm{g}$ of total RNA mixed with 20 pmol oligo $(\mathrm{dT})_{15}$ primer $\mathrm{T} 15$ was treated at $70^{\circ} \mathrm{C}$ for $10 \mathrm{~min}$ and then cooled on ice for $2 \mathrm{~min}$. After adding $2 \mu \mathrm{l}$ of $10 \times$ buffer, $20 \mathrm{nmol}$ dNTP mixture, $0.2 \mu \mathrm{mol}$ DTT, 40 units placental RNase inhibitor, and 100 units reverse transcriptase $\mathrm{M}-\mathrm{MLV}$, the reaction system was made up to $20 \mu \mathrm{l}$ volume in the end using DEPC- $\mathrm{ddH}_{2} \mathrm{O}$. Then it was incubated at $42^{\circ} \mathrm{C}$ for $1 \mathrm{~h}$, and the reaction was terminated by incubation at $95^{\circ} \mathrm{C}$ for $5 \mathrm{~min}$.

\section{Amplification of MSBPase cDNA fragments}

The degenerated PCR primers (forward primer: SBP1, reverse primer: SBP-2) were designed according to the high conserved domain of the amino acid sequences of SBPases in GenBank. The total volume of the PCR reaction mixture was $25 \mu \mathrm{l}$, containing $1 \mu \mathrm{l}$ of the first strand cDNA, $0.5 \mu \mathrm{M}$ of each primer, $2.5 \mu \mathrm{l}$ of $10 \times$ PCR buffer, $0.4 \mathrm{mM}$ dNTPs and 1 unit of Taq DNA polymerase. The reaction was denatured at $94^{\circ} \mathrm{C}$ for $5 \mathrm{~min}$, and then followed by 35 cycles of $1 \mathrm{~min}$ at $94^{\circ} \mathrm{C}, 1 \mathrm{~min}$ at $56^{\circ} \mathrm{C}$ and $1 \mathrm{~min}$ at $72^{\circ} \mathrm{C}$, finally extended for $10 \mathrm{~min}$ at $72{ }^{\circ} \mathrm{C}$. The PCR products were separated on a $1 \%$ agarose gel. The bands of the expected size were purified using DNA Gel Extraction Kit.

\section{Rapid amplification of the $3^{\prime}$-and $5^{\prime}$-end of MSBPase cDNAs}

RACE-PCR was used to amplify the 3 '-and 5 '-end SBPase cDNAs. On the basis of the obtained sequence information, specific primers were designed and nested PCR was performed for $3^{\prime}$-and 5'-RACE. For 3'-RACE, the first strand cDNA synthesized by primer T15 was used as template. For 5'-RACE, the first strand cDNA synthesized by T15 was further tailed poly (C) at the 3 '-end with terminal deoxynucleotidyl transferase according to the manufacturer instructions, and the poly (C)-tailed cDNA was used as template. The first round PCR was carried out using a forward specific primer 3SW designed against obtained sequence information by RT-PCR and a reverse adapter primer T15 for 3'-RACE, and using primer G16 and the reverse specific primer $5 \mathrm{SW}$ for 5'-RACE. The second round PCR was performed using a forward specific primer 3SN and the adapter primer ST for $3^{\prime}$-RACE, and using primer GT and the reverse specific primer $5 \mathrm{SN}$ for $5^{\prime}$-RACE, with the first round PCR products as templates, respectively. The two round amplification reactions were carried out as described in RT-PCR. The bands of the expected size were purified using DNA Gel Extraction Kit.

\section{Cloning and sequencing}

All the DNA fragments obtained from RT-PCR and RACE-PCR were subcloned individually into the pMD18-T vector. After transformation, the positive clones were selected and further sequenced by Shanghai Invitrogen Biological Engineering Technology and Service Co., Ltd.

\section{Deducing of the Full-length MSBPase cDNA}

By comparing the overlapping sequences of the partial cDNA fragments, the full-length cDNA sequence was deduced and cloned by RT-PCR.

\section{Molecular information analysis}

The deduced amino acid sequence of MSBPase was aligned with other plant SBPases using DNAMAN multiple alignments program. Homology analysis was performed with BLASTP programs on NCBI (http://www. ncbi.nlm.nih.gov/BLAST). The molecular weight and isoelectric point were predicted with Compute $\mathrm{pI} / \mathrm{Mw}$ tool (http://www.expasy.org/tools/pi_tool.html). Prediction of the structure of a N-terminal extension in MSBPase was performed by using TargetP 1.1 Server (http://www.cbs. dtu.dk/services/TargetP). The structure of MSBPase domain was analyzed with NCBI database (http://www. ncbi.nlm.nih.gov/Structure).

\section{Results and Discussion}

\section{Cloning of the Full-length cDNA of MSBPase}

Based on the primer designed for the amplification of the partial cDNA fragment of MSBPase, an expected $510 \mathrm{bp}$ cDNA fragment was amplified (Fig. 1 A). The

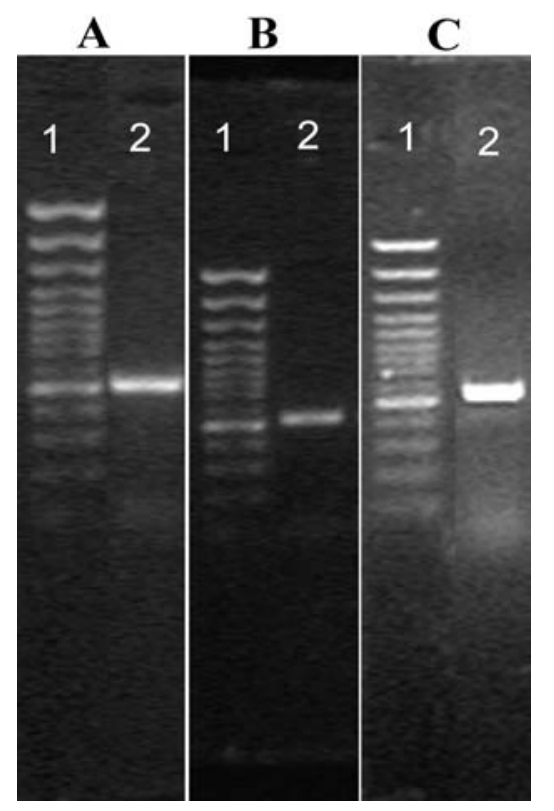

Figure 1 A. - PCR amplification of the fragment of MSBPase cDNA; B: PCR amplification of 3'-terminal sequence of MSBPase cDNA; C: PCR amplification of 5 -terminal sequence of MSBPase cDNA; 1: DNA marker 100 bp; 2: PCR amplification band. 
nucleotide sequence of this fragment showed a high similarity with the previous cloned SBPase genes from other plant species. By two rounds of nested PCR, a 540 bp DNA fragment of the 3'-RACE (Fig. 1 B) and a 600 bp DNA fragment of the 5'-RACE (Fig. $1 C$ ) were amplified, respectively. By comparing the overlapping sequences of the partial cDNA fragments, the full-length cDNA sequence of MSBPase was obtained (GenBank accession number DQ995346). The 1,527 bp full-length cDNA contained a 1,179 bp ORF beginning with the initiation codon ATG at nucleotide 133 and terminating with stop codon TAA at nucleotide 1309 (Fig. 2). The ORF of MSBPase included 393 amino acids, whose predicted molecular weight was $42.6 \mathrm{kDa}$ and isoelectric point was 5.85 (Fig. 2).

\section{Nucleotide and deduced amino acid sequence analysis of} MSBPase

To obtain more information about the nucleotide and deduced amino acid sequence of MSBPase, the sequence comparisons as well as the multiple alignment analysis were carried out via blast search in GenBank database (http://www.ncbi.nlm.nih.gov/BLAST). The results revealed that there was high homology between the MSBPase and SBPases in many other plant species. MSBPase deduced amino acid sequence shared $83 \%$, $81 \%, 75 \%$ and $77 \%$ identity with its counterparts from Arabidopsis thaliana, Spinacia oleracea, Triticum aestivum and Oryza sativa, respectively (Fig. 3). The highly conserved amino acid region of SBPase implies that these amino acids may play an important role. Though limited homology exists in the N-terminal, the general features of chloroplast transit peptide could be observed based on rich Ser and absent acidic residues (FUJIMORI et al., 1998; Von-HEIJINE et al., 1989). Moreover, the structure prediction of the N-terminal of MSBPase also indicated that it contained the putative chloroplast transit peptide of 55 amino acids, which directs the protein translocation from cytosol to chloroplast. Therefore, after the putative transit peptide removed, the molecu-
1

61 121

181

17

241

37

301

57

361

77

421

97

421

117

541

137

601

157

661

177

721

197

781

217

841

237

901

257

961

277

1021

297

1081

317

1141

337

1201

357

1261

377

1321

13 B 1

1441

1501
AAACCAATTACTAGTAGTCTAGTAGTACAAGCAAAGTAAGCAAATAGAGCTTTTGAAGAA ATCAAAGCTCTTCCTTGGTGTTTTTTTTTTTTGGTGTTAAT TTGAGGAAAAAATTTTCGA CATAATTCATTATGGAGACAAGTATCACTTGCTACACTCGTGGAGCTTTCCTTCCTAAT $\begin{array}{lllllllllllllllll}M & E & T & S & I & T & C & Y & T & R & G & A & F & L & P & N\end{array}$ ATCTCGTCTTCGCATTCCATGTCTTTTGTGTCTCCCTCATCCATCTCTGCATCTTTCAAC

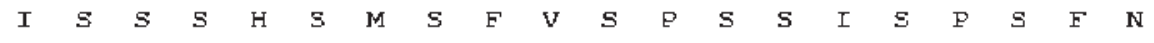
TCCAAGATTCTGAAATCGAGCTCGCTGTTCGGGGAATCATTACGCACGGTGCCAAAATCT $\begin{array}{llllllllllllllllllll}S & \mathbb{R} & \mathrm{I} & \mathrm{L} & \mathrm{K} & \mathrm{S} & \mathrm{S} & \mathrm{S} & \mathrm{L} & \mathrm{F} & \mathrm{G} & \mathrm{E} & \mathrm{S} & \mathrm{L} & \mathrm{R} & \mathrm{T} & \mathrm{V} & \mathrm{F} & \mathrm{K} & \mathrm{S}\end{array}$ TGTCTAAAGGTTTCAAAATCGAAGAACGGT TTGCCAGTGACGAAATGGGAGATAGGCGAA $\begin{array}{lllllllllllllllllllll}S & L & K & V & S & K & S & K & N & A & L & E & V & T & K & C & E & I & G & E\end{array}$ AGCTTGGAGGAATTCCTTATAAAGGGGAGGCCTGATAAAGGACTGAGGGCATTGCTGATC $\begin{array}{llllllllllllllllllll}5 & \mathrm{~L} & \mathrm{E} & \mathrm{E} & \mathrm{F} & \mathrm{L} & \mathrm{I} & \mathrm{K} & \mathrm{A} & \mathrm{T} & \mathrm{F} & \mathrm{D} & \mathrm{K} & \mathrm{G} & \mathrm{L} & \mathrm{R} & \mathrm{A} & \mathrm{L} & \mathrm{I} & \mathrm{I}\end{array}$ TCGATGGGAGAAGCATTGAGGACCATTGCCTTTAAAGTGAGAACAGCTTCTTGTGGTGGA $\begin{array}{llllllllllllllllllll}S & M & G & E & A & L & R & T & I & A & F & K & V & R & T & A & S & C & G & G\end{array}$ ACGGCTTGTGTCAATTCOTTTGGTGACGAACAGCTGGCAGTTGATATGCTTGCAAACAAC

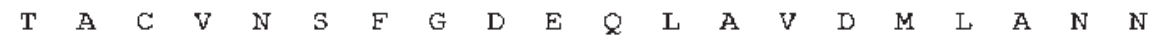
CTICTTTTTGAGGCCCTAAGGTACTCCCATTTCTGCAAGTATGCTTGTTCCGAGGAAGTT

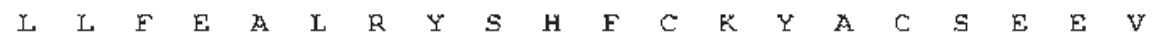
CGTGAGCTCCAAGACATGGGAGGCCCAGTT GAAGGTGGATTCAGTGTGGCTTTCGATCCC $\begin{array}{llllllllllllllllllll} & E & I & Q & D & M & G & G & F & V & E & G & G & F & S & V & A & F & D & P\end{array}$ CTCGACGITTCTAGCATCGTCGACACAAAT T TCACGGTGGGGACAAT T TTGGAGTGTGG

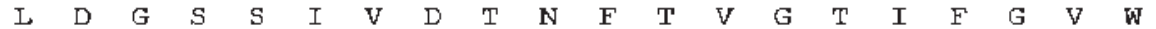
CCAGGAGATAAGTTGACTGGAGTAACAGGARGAGACCAAGTTGCTGCTGCCATGGGGATT $\begin{array}{llllllllllllllllllll}P & G & D & K & I & T & G & V & T & G & \mathbb{R} & D & \& & V & A & A & A & M & G & I\end{array}$ TATGGCCCTAGAACTACATATGTTCTTGCTCTTAAAGACGTTCCCGGGACACACGAATTC $\begin{array}{lllllllllllllllllllllll}Y & G & F & R & T & T & Y & Y & L & A & L & K & D & V & F & G & T & H & E & F\end{array}$ CTTCTTTGGATGAAGGAAAATGGCAGCACGTCAAGGAGACAACAGAAATTGGTGAGGGA $\begin{array}{llllllllllllllllllll}L & L & L & D & E & G & K & W & Q & H & V & K & E & T & T & E & I & G & E & G\end{array}$ AAGCTTTTCTCTCCTGGAAACT TGAGAGCCACTTTTGATAATCCGGAATACAACAAGCTA $\begin{array}{llllllllllllllllllll}K & L & F & S & P & G & N & I & R & A & T & E & D & N & F & E & Y & \mathbb{N} & K & L\end{array}$ ATTGATTACTATGTGAAAGAGAAGTACACTTTGAGATACAGTGGAGGAAGGGTACCCGAC

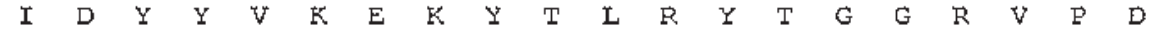
GTCAACCAGATTATTGTGAAAGAGAAGGGTATTTCACCAATGTCTTATCCCCATCAGCA

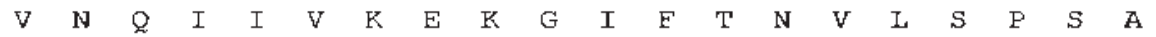
AAGGCCAAGCTAAGACTGTTGTTTGAGGTGGCCCCACTTGGTTTCTTGATTGAGAAGGCA $\begin{array}{lllllllllllllllllllll}K & A & K & L & R & L & L & F & E & V & A & B & L & G & F & L & I & E & K & A\end{array}$ GGAGGGTTCAGTAGTGATGGCCATCAGTCTGTGCTAGAAAAGGTGATTGAGAACCTTGAT $\begin{array}{llllllllllllllllllll}G & G & F & 5 & 5 & \mathrm{~J} & G & \mathrm{H} & Q & 5 & \mathrm{~V} & \mathrm{~L} & \mathrm{E} & \mathrm{K} & \mathrm{V} & \mathrm{I} & \mathrm{E} & \mathrm{N} & \mathrm{I} & \mathrm{D}\end{array}$ GATAGAACTCAGGTTGCGTACGGATCGAAAAACGAGATTATCCGATTCGAAGAAACTCTA $\begin{array}{llllllllllllllllllllllll}D & R & T & Q & V & A & Y & G & S & K & N & E & I & I & R & F & E & E & T & L\end{array}$ TACGGATCTTCCAGGCTCAAGGGAGCAGTGCOTGTTGGAGCTGCTGCATAAATATTACAG

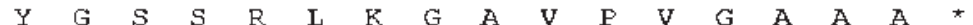

AACTACATGTGATTCTTACTTATGTTTGTAGCTCCTTTCTTTTGTTTTTGTTTTACTTG GTTTTCTCCTCAAGTAAAAATT TAAAAAATGCTTGATATTCATAGTCTGGCGAGCTGTGT ATCATAGTGCATAATGGGAGGCTTGAAATTAAGACTTTTGAATCATCACAAGGTTGAATC TT TCTCTGCTCTAAAAAAAAAAAAAAA

Figure 2. - Nucleotide sequence of MSBPase cDNA clone and deduced amino acid sequence. The amino acid sequence of the polypeptide is given in a three-letter code for each residue. Potential codons for initiation (ATG) and termination (TAA) of translation are boxed. 


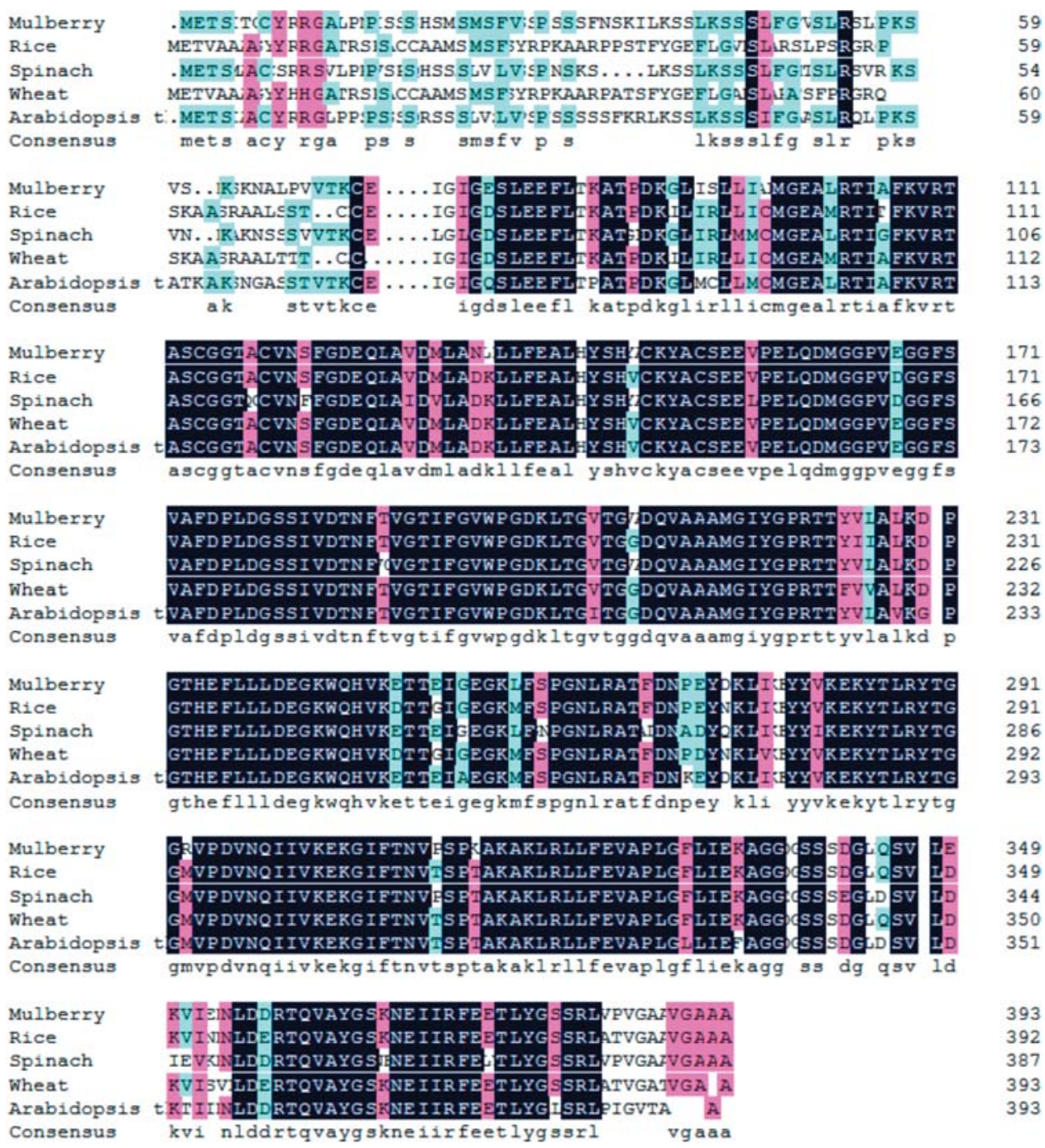

Figure 3. - Alignment of the amino acid sequence of MSBPase with that of Arabidopsis thaliana (AAM91137), spinach (Spinacia oleracea) (AAB81104), wheat (Triticum aestivum) (CAA46507) and rice (Oryza sativa) (AA022559) SBPases. Amino acid sequences were downloaded from the NCBI database and the alignments were performed using the DNAMAN program. Identical amino acids were black shaded and similar amino acids were gray shaded.

lar weight of predicted mature protein is in the range of 35-36 kDa which is in good agreement with earlier biochemical studies about SBPase (NishizAwA and BuChanAN, 1981; CADET et al., 1987).

\section{Phylogenetic and molecular evolutionary analysis}

To investigate the molecular evolutionary relationships among different species, a phylogenetic tree was constructed based on the amino acid sequences of MSBPase, SBPase and FBPase from various organisms including plants, fungi, insects and animals by performing blast search in GenBank database (http://www.ncbi. nlm.nih.gov/BLAST/). As expected, the result showed that the MSBPase gene fell into the plant group (Fig. 4). Interestingly, all the SBPase genes were derived from a common ancestor in evolution, suggesting that they shared a common evolutionary origin. In addition, the amino acid sequences of SBPases and FBPases from various species shared $28-32 \%$ identical residues, indicating that the two enzymes may share a common cat- alytic mechanism and evolution origin, and acquire substrate specificity following divergence from a bifunctional enzyme (Christine et al., 1992; HANNAERT et al., 2003). Recently, it is reported that the evolutionary histories of the SBPase and FBPase gene families are very complex, including extensive paralogy, lateral transfer, and retargeting between cellular compartments (ROGERS and KeELiNG, 2004)

\section{The 3-D structure of MSBPase}

The 3-D structure of MSBPase was determined by aligning the amino acid sequences obtained from the NCBI database. It was clear from initial sequence comparisons of SBPases and FBPases from various sources that these two enzymes were structurally related. The structural similarities between SBPase and FBPase highlighted by these amino acid sequence comparisons made it possible to gain an insight into the structure of SBPase by the 3-D structure model of FBPase (HANNAERT et al., 2003). The model (Fig. 5) showed that 


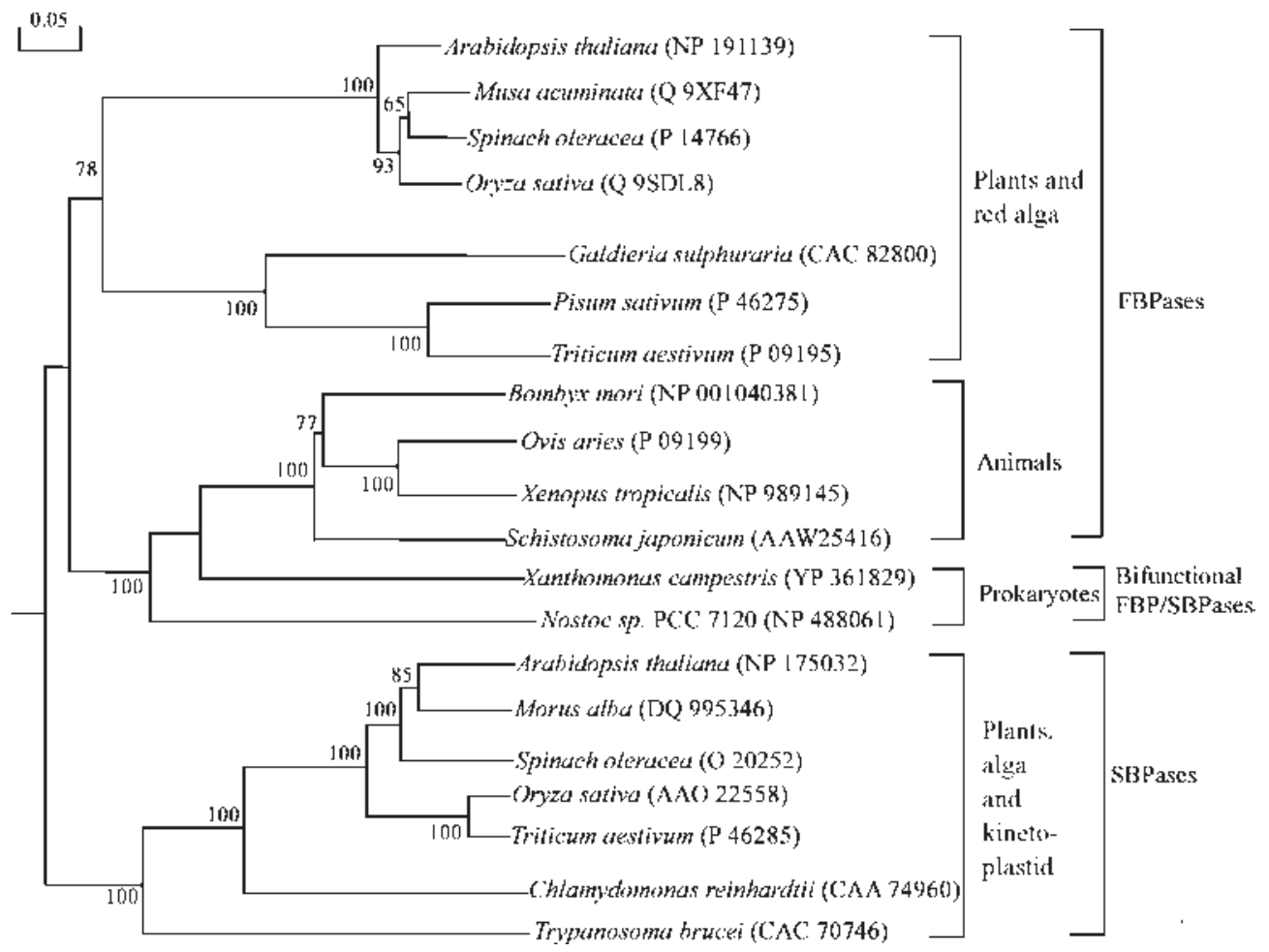

Figure 4. - Neighbor-joining tree of SBPases and FBPases. The SBPase and FBPase sequences were compared by performing blast search in GenBank database (http://www.ncbi.nlm.nih.gov/ BLAST).

MSBPase contained two subunits and each subunit was composed of four $\alpha$-helices and six $\beta$-sheets joined by turns and loops and folded into a hexahedron shape. There are four cysteine residues apart in a predicted loop between two $\alpha$-helices, two of which probably form a disulphide bond when oxidized analogous to the allosteric regulation of FBPase by adenosine monophosphate in terms of the resulting structural changes. Active sites are located at the interface between pairs of

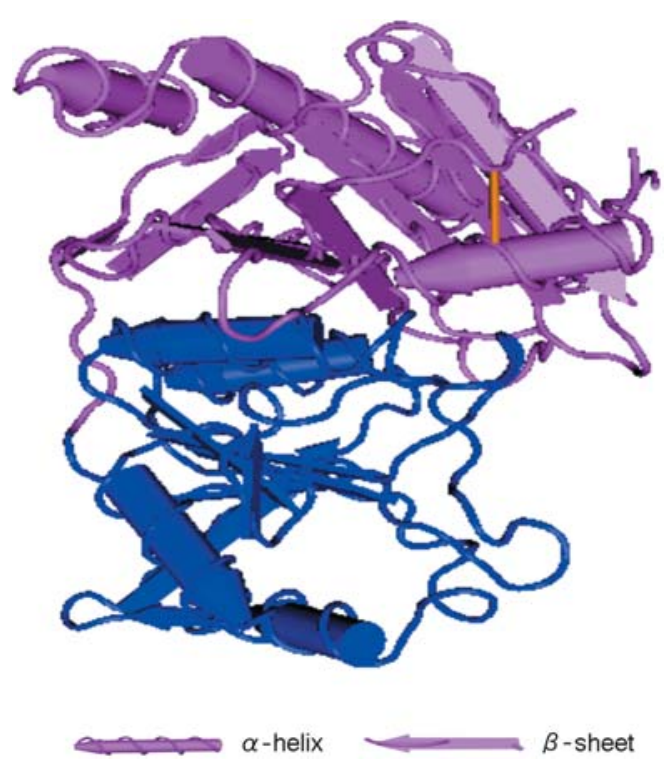

Figure 5. - Predicted tridimensional structure of MSBPase. The homodimer pair of subunits which constitute MSBPase are marked in red and blur colors. subunits, with each subunit contributing to the active site of its partner (DUNFORD et al., 1998).

In conclusion, we have isolated and characterized a new SBPase cDNA clone from mulberry. Evolution analysis revealed that the MSBPase gene had high homology with other plant SBPases. SBPases and FBPases share a common evolution origin and are structurally related. To our knowledge, this is the first report of the molecular studies of SBPase from mulberry. Further investigation should be carried out focusing on the biochemical and physiological function of MSBPase.

\section{References}

ANJA, S., B. MARco and H. Riger (2002): Overexpression of the potential herbicide target sedoheptulose-1,7-bisphosphatase from Spinacia oleracea in transgenic tobacco. Mol. Breed. 9: 53-61.

Cadet, F., J. C. Meunier and N. Ferte (1987): Isolation and purification of chloroplastic spinach (Spinacia oleracea) sedoheptulose-1,7-bisphosphatase. Biochem. J. 241: 71-74.

Chang, S., J. Puryear and J. CAiney (1993): A simple and efficient method for RNA isolating from pine trees. Plant Mol. Biol. Rep. 11: 113-116.

Christine, A. R., C. L. Julie, M. W. Nicola, P. Susan and A. D. TRIstan (1992): cDNA and gene sequences of wheat chloroplast sedoheptulose-1,7-bisphosphatase reveal homology with fructose-l,6-bisphosphatases. Eur. J. Biochem. 205: 1053-1059.

Christine, A. R., C. L. Julie and A. D. Tristan (1999): New insights into the structure and function of sedoheptulose-1,7-bisphosphatase; an important but neglected Calvin cycle enzyme. J. Exp. Bot. 50: 1-8. 
Dunford, R. P., M. C. Durrant, M. A. Catley and T. A DYER (1998): Location of the redox-active cysteines in chloroplast sedoheptulose-1,7-bisphosphatase indicates that it is allosteric regulation is similar but not identical to that of fructose-1,6-bisphosphatase. Photosynth. Res. 58: 221-230.

FujImori, K., S. TADA, S. KANAI and D. OHTA (1998): Molecular cloning and characterization of the gene encoding $\mathrm{N}^{\prime}$-[(5'-phosphoribosyl)-formimino]-5-aminoimidazole-4carboxamide ribonucleotide (BBM II) isomerase from Arabidopsis thaliana. Mol. Gen. Gent. 259: 216-223.

Geiger, D. R. and J. C. Servaites (1995): Diurnal regulation of photosynthetic carbon metabolism in C3 plants. Annu. Rev. Plant Physiol. Plant Mol. Biol. 45: 235-56.

Hannaert, V., E. SaAvedra, F. Duffieux, J. P. Szikora, D. J. Rigden, P. A. Michels and F. R. OpPERdoes (2003): Plant-like traits associated with metabolism of Trypanosoma parasites. Proc. Natl. Acad. Sci. USA 100: 1067-1071.

Jain, A. K., R. M. Vincent and C. L. Nessler (2000): Molecular characterization of a hydroxymethylglutarylCoA reductase gene from mulberry (Morus alba L.). Plant Mol. Biol. 42: 559-569.
NishizaWA, A. N. and B. B. Buchanan (1981): Enzyme regulation in $\mathrm{C} 4$ photosynthesis. Purification and properties of thioredoxin-linked fructose bisphosphatase and sedoheptulose bisphosphatase from corn leaves. J. Biol. Chem. 256: 6119-6126.

Petterson, G. and U. Ryde-Petterson (1989): Dependence of the Calvin cycle activity on kinetic parameters for the interaction of non-equilibrium cycle enzymes with their substrates. Eur. J. Biochem. 186: 683-687.

Poolman, M. G., D. A. Fell and S. Thomas (2000): Modeling photosynthesis and its control. J. Exp. Bot. 51: 319-328.

Rogers, M. and P. J. KEELING (2004): Lateral transfer and recompartmentalization of Calvin cycle enzymes of plants and algae. J. Mol. Evol. 58: 367-375.

Von-Heijine, G., J. Steppuhn and R. G. HeRRMAnN (1989): Domain structure of mitochondrial and chloroplast targeting peptides. Eur. J. Biochem. 180: 535-545.

WOODROW, I. E. and J. A. BERRY (1988): Enzymic regulation of photosynthetic $\mathrm{CO}_{2}$ fixation in C3 plants. Ann. Rev. Plant Physiol. Plant Mol. Biol. 39: 533-594.

\title{
Genetic Diversity and Population Structure of Apricot (Prunus armeniaca L.) from Northern Pakistan using Simple Sequence Repeats
}

\author{
By M. Ali Khan ${ }^{1)}$, F. Maghuly ${ }^{2)}$, E. G. Borroto-Fernandez ${ }^{2)}$, A. Pedryc $^{3)}$, H. Katinger ${ }^{2)}$ and M. LaimeR ${ }^{2), 4)}$
}

(Received 19 $9^{\text {th }}$ December 2006)

\begin{abstract}
A collection of $44 P$. armeniaca accessions and three related species, from diverse geographic areas, covering the entire Northern hemisphere with emphasis on Pakistani apricots, was screened with 10 SSR primer pairs developed in apricot, to characterize the cultivars and establish their genetic relationship. Given the fact that the Central Asian region is considered a center of origin of apricot, particular attention was devoted to accessions from the Hunza region of Pakistan. The primers correctly amplified a repeatable polymorphic pattern, which

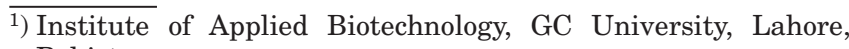
Pakistan.

2) Plant Biotechnology Unit, Institute of Applied Microbiology BOKU, Muthgasse 18, A-1190 Vienna, Austria.

${ }^{3}$ ) Corvinus University, Villányi ut 29-43, 1118 Budapest, Hungary.

4) Corresponding author: MARGIT LAIMER. University of Natural Resources and Applied Life Sciences, Institute of Applied Microbiology, Plant Biotechnology Unit, Nussdorfer Lände 11, 1190 Vienna, Austria. Tel. +43-1-36006 6560, Fax +43-1-3697615, Email: m.laimer@iam.boku.ac.at
\end{abstract}

unequivocally distinguished all genotypes under study. Altogether 123 alleles were identified with an average of 12.30 alleles per locus. The observed heterozygosity for individual loci ranged from 0.28 to 0.77 with an average of 0.64. A neighbour joining method identified four groups from: (A) Central Asia, (B) Irano-Caucasia, (C) Continental Europe and (D) North America. The dendrogram confirms the historic dissemination pathways of apricot from its centre of origin in Asia to the West. Apricot cultivars from the Hunza region (Northern Pakistan) revealed a high variability, as genetic diversity is still conserved due to the traditional practice of planting seeds from the best trees.

Key words: Genetic variation, microsatellites, eco-geographic groups, Hunza, center of origin.

\section{Introduction}

Botanists distinguish the following apricot species: $P$. ansu, $P$. armeniaca, $P$. brigantina, $P$. mandshurica, $P$. $x$ dasycarpa, $P$. holosericea, $P$. mume and $P$. siberica (OECD, 2002). Most cultivated apricots, belong to the species $P$. armeniaca L., are native to Asia and Caucasus 